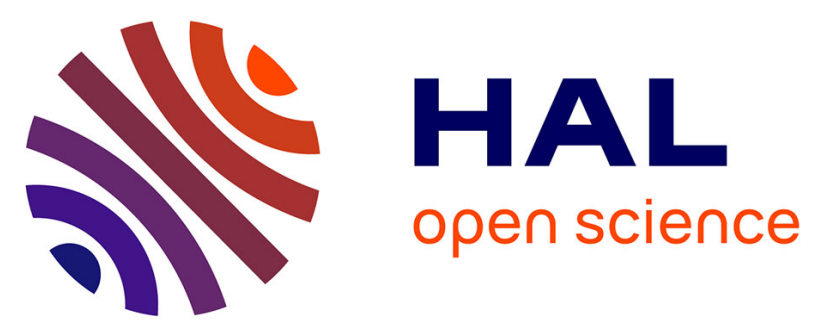

\title{
SUIVI AUTOMATISÉ DES CRUES À FORT TRANSPORT SOLIDE DANS LES TORRENTS : STRATÉGIE DE MESURE ET POTENTIEL DES DONNÉES COLLECTÉES
}

Firmin Fontaine, Coraline Bel, Hervé Bellot, Guillaume Piton, Frédéric Liébault, Marie Juppet, Kristian Royer

\section{To cite this version:}

Firmin Fontaine, Coraline Bel, Hervé Bellot, Guillaume Piton, Frédéric Liébault, et al.. SUIVI AUTOMATISÉ DES CRUES À FORT TRANSPORT SOLIDE DANS LES TORRENTS : STRATÉGIE DE MESURE ET POTENTIEL DES DONNÉES COLLECTÉES. Collection EDYTEM. Cahiers de géographie, 2017, Monitoring en milieux naturels - Retours d'expériences en terrains difficiles, 19, pp.213-219. 10.3406/edyte.2017.1385 . hal-01656535

\section{HAL Id: hal-01656535 \\ https://hal.science/hal-01656535}

Submitted on 7 Dec 2017

HAL is a multi-disciplinary open access archive for the deposit and dissemination of scientific research documents, whether they are published or not. The documents may come from teaching and research institutions in France or abroad, or from public or private research centers.
L'archive ouverte pluridisciplinaire HAL, est destinée au dépôt et à la diffusion de documents scientifiques de niveau recherche, publiés ou non, émanant des établissements d'enseignement et de recherche français ou étrangers, des laboratoires publics ou privés. 


\title{
SUIVI AUTOMATISÉ DES CRUES À FORT TRANSPORT SOLIDE DANS LES TORRENTS STRATÉGIE DE MESURE ET POTENTIEL DES DONNÉES COLLECTÉES
}

\author{
SEDIMENT-LADEN FLOW MONITORING IN TORRENTS: \\ MEASUREMENT STRATEGIE AND COLLECTED DATA POTENTIAL \\ Firmin FONTAine ${ }^{1}$, Coraline BEL ${ }^{1}$, Hervé BELLOT ${ }^{1}$, GuIllaume PITON $^{1}$, \\ FrÉDÉRIC LIEBAULT ${ }^{1}$, MARIE JUPPET ${ }^{2}$, KRISTIAN ROYER $^{2}$ \\ ${ }^{1}$ Univ. Grenoble Alpes, IRSTEA, UR ETNA, 2 rue de la papeterie, 38402 St Martin d'Hères. \\ ${ }^{2}$ ONF-RTM, Services de l'Isère et des Alpes Maritimes. \\ Contact :firmin.fontaine@irstea.fr
}

\begin{abstract}
RÉSUMÉ
Dans les vallées de montagne urbanisées, les crues des torrents représentent un risque marqué. La mesure in situ automatisée permet d'une part de détecter l'occurrence d'un événement et d'autre part d'améliorer la compréhension de ces phénomènes. Ce suivi instrumenté se caractérise également par des contraintes fonctionnelles fortes, imposées par un environnement de mesure difficile. Ce chapitre présente l'intérêt de cette approche, la stratégie de mesure adoptée et le potentiel des données recueillies par ces dispositifs à des fins opérationnelles et de recherche.
\end{abstract}

MotS-CLÉS : SUIVI INSTRUMENTE, TORRENT, LAVE TORRENTIELLE, CHARRIAGE, GÉOPHONES.

\begin{abstract}
In urbanized mountainous regions, torrent-related hazards may engender substantial risks. In-situ measurement, by means of monitoring station, makes it possible to (i) detect event occurrence and (ii) improve the current understanding of such phenomena. However, torrent monitoring is constrained by harsh operating conditions. This paper aims at highlighting the benefits of such an approach, the adopted measurement strategy, and the potential of collected data set for both operational and research purposes.
\end{abstract}

KEYWORDS: MONITORING, TORRENT, DEBRIS FLOW, BEDLOAD, GEOPHONES.

\section{INTRODUCTION}

Les crues dans les torrents peuvent mobiliser de grandes quantités de sédiments par charriage ou sous forme de lave torrentielle. Ces phénomènes étant capables de couper les routes, de déposer d'importants volumes de débris sur les cônes de déjection et de détruire ouvrages et bâtis (e.g., crue à Modane (73) en août 2014 ; crue à Barèges (65) en juin 2013 : Figure 1), ils représentent un risque majeur dans les vallées habitées. La plupart des torrents les plus actifs sont situés en terrains domaniaux. Les services RTM (Restauration des Terrains de Montagne) de l'ONF (Office National des Forêts), acteurs majeurs de la prévention et de la gestion des aléas torrentiels, organisent (i) la mise en place et la maintenance d'ouvrages de correction torrentielle et (ii) le recensement des crues torrentielles dans une base donnée événementielle (rtm-onf.ifn.fr).
En parallèle à ce travail, ces phénomènes font l'objet de recherches scientifiques - au moyen d'observatoires de terrain, d'approches expérimentales et de simulations numériques - qui visent à améliorer la compréhension des processus physiques qui les gouvernent et des interactions entre ces processus et leur environnement (Recking et al., 2013). Les approches numériques et de modélisation physique manquent encore de bonnes observations de terrain, à même de constituer des cas d'études pour la validation et l'amélioration des modèles. La multiplication d'observations in situ des crues est nécessaire pour rendre ces approches plus pertinentes et robustes.

D'un point de vue scientifique, le monitoring fournit des informations, à la fois qualitatives et quantitatives, sur les processus en jeu lors des crues à fort transport 
solide (e.g., dynamique de l'initiation et de la propagation des fronts de lave torrentielle). D'un point de vue opérationnel, le monitoring d'un torrent permet de connaître son activité en temps réel. Cette information peut s'avérer intéressante dans le cadre d'un processus d'aide à la décision (Plan Communal de Sauvegarde), ou d'un dispositif d'alerte automatique (feux routiers, alarme). À plus long terme, les données collectées pourraient également venir enrichir des bases de données telle que celle du RTM, et ainsi améliorer la connaissance historique et les données d'entrées des modèles de zonage du risque et de dimensionnement d'ouvrages.

Depuis une vingtaine d'années, de nombreuses initiatives ont été prises pour développer la mesure in situ des crues torrentielles (e.g., Badoux et al., 2009). À la complexité des phénomènes observés viennent s'ajouter des contraintes fonctionnelles fortes. Le caractère très rapide des crues impose l'utilisation de systèmes autonomes et réactifs. Ces contraintes temporelles s'ajoutent également à des contraintes matérielles de difficulté d'accès, de manque d'alimentation énergétique et de risque de destruction des capteurs intrusifs. Depuis 2010, l'unité ETNA du centre IRSTEA de Grenoble développe et gère des stations de monitoring dédiées aux écoulements dans les torrents (Navratil et al., 2011). Cet article détaille dans un premier temps la stratégie de mesure des dispositifs expérimentaux, fondée en partie sur l'utilisation de capteurs sismiques. Dans un second temps, il met en avant le potentiel des données recueillies par ces dispositifs à des fins opérationnelles et de recherche.

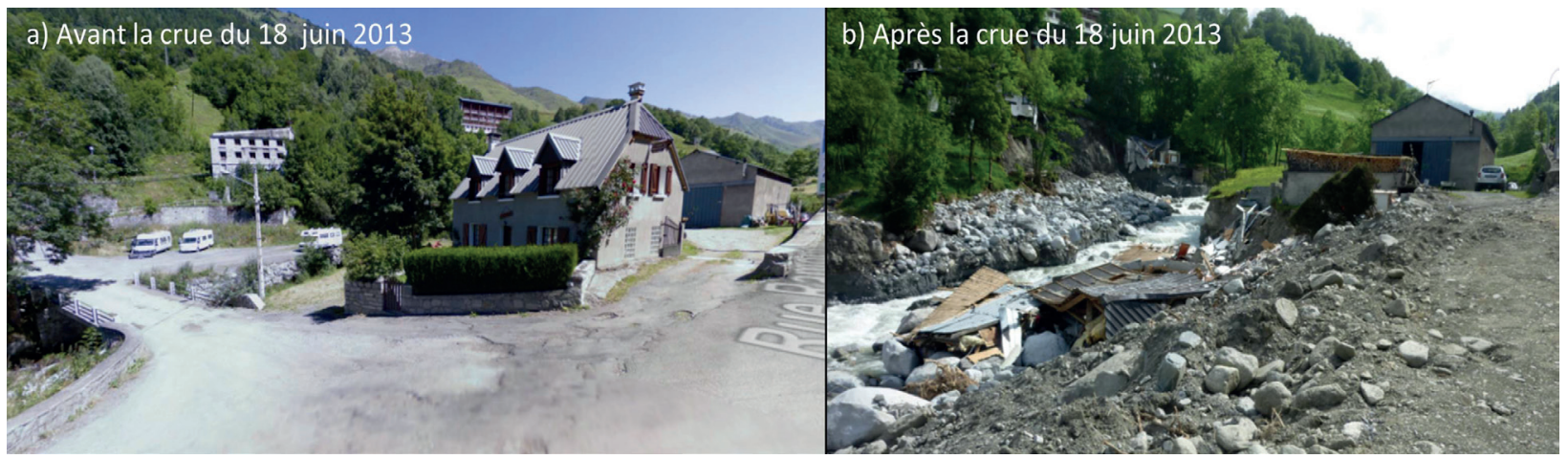

Figure 1 - Crue du Bastan à Barèges (65). a) Avant la crue (CGooglestreetmap), et b) après la crue du 18 juin 2013 (Photo : G. PITON). La maison au premier plan, ainsi que le pont sur la gauche et le parking attenant ont tous été emportés par la crue.

\section{MATÉRIEL ET MÉTHODE}

\section{Stratégie de mesure}

Hors cas particuliers des débâcles glaciaires et des ruptures d'ouvrages hydrauliques, les précipitations sont généralement responsables de l'initiation des crues torrentielles. La pluviométrie est donc un paramètre critique à mesurer pour caractériser les conditions d'occurrence. Le passage d'une crue à fort transport solide (charriage ou lave torrentielle) va générer des vibrations sismiques. En complément de prises de vues et d'une mesure de niveau, il apparaît donc pertinent de mesurer les vibrations à proximité du chenal d'écoulement à l'aide de géophones pour détecter ces épisodes de transport et les caractériser. Un dispositif type est présenté en Figure 2. D'autres capteurs peuvent être utilisés dans le cadre du monitoring en milieu torrentiel ont fait l'objet d'une synthèse publiée récemment sur le sujet (Cavalli et al., 2013).

Une mesure in situ en continu implique plusieurs contraintes fortes : (i) la gestion d'un volume de données important, (ii) la source d'énergie limitée fournie par une alimentation solaire nécessaire pour un système autonome, et (iii) le caractère difficile de l'environnement de mesure (humidité, risque d'arrachement des capteurs). Pour répondre à ces contraintes, nous avons fait le choix d'utiliser des centrales d'acquisition environnementales (CR1000 Campbell®). Ce type de dispositif, très durci, consomme peu d'énergie (20 mA). Il permet d'interfacer, à la fois, des capteurs variés et des systèmes de communication à distance, essentiels pour optimiser le suivi du site et la maintenance de la station. Son fonctionnement s'apparente à une boucle durant laquelle il est possible à la fois de mesurer, d'interpréter et de sauvegarder des informations fournies par les capteurs (Figure 3).

Les principes de mémoire circulaire et de stockage événementiel sont utilisés pour sauvegarder les données à une fréquence de $10 \mathrm{~Hz}$. Cela permet d'augmenter sensiblement la capacité de stockage du dispositif tout en évitant de manquer le début d'une crue soudaine. Pour les prises de vues, un système de seuil basé sur l'intensité de pluie a été mis en place pour déclencher de manière événementielle les appareils photos. Ainsi, la station présente une autonomie de plusieurs mois à un an en fonction du nombre de crues qui se produisent. 

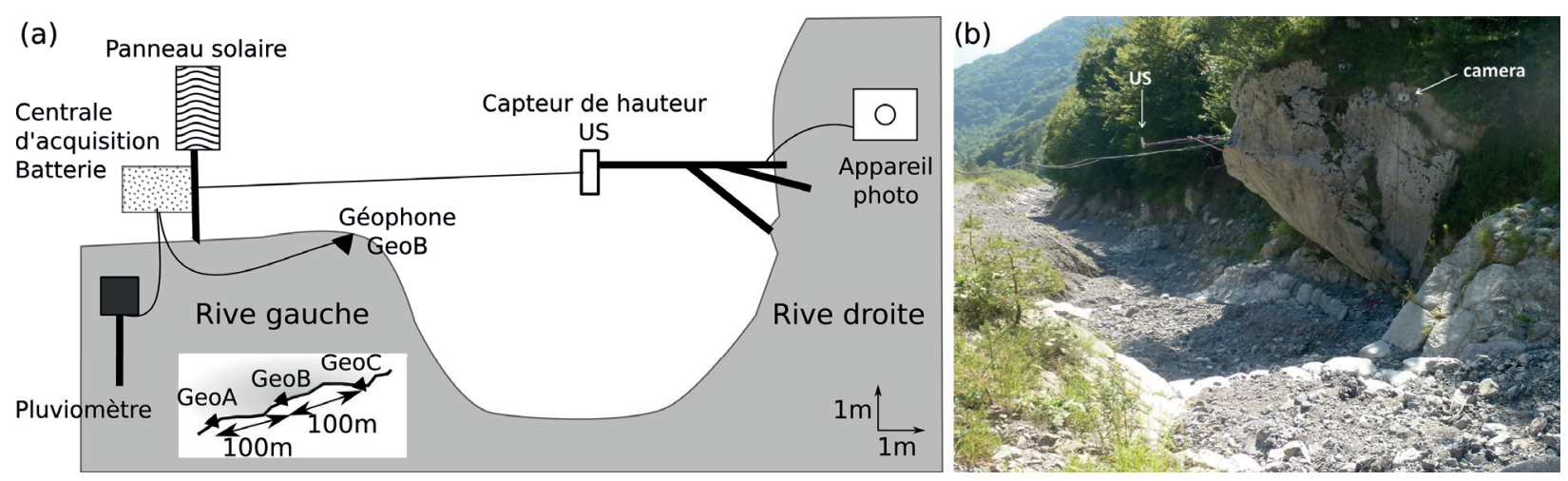

Figure 2 - Exemple d'implantation d'une station de monitoring au droit d'un barrage de correction torrentielle. a) Schéma de principe. b) Photo de la station du Manival (38) vue vers l'aval.

\begin{tabular}{|c|c|c|}
\hline Mesures & $\begin{array}{c}\text { Scan rapide }(100 \mathrm{~ms}) \\
\text { Tâches }\end{array}$ & Enregistrement \\
\hline $\begin{array}{l}\text { - Activité sismique } \\
\text { - Hauteur d'écoulement } \\
\text { - Précipitation } \\
\text { - État de la centrale (ex: niveau de batterie) }\end{array}$ & \begin{tabular}{|l|} 
- Calcul du cumul de pluie \\
- Passage ou non en mode événement \\
- Asservissement des prises d'images
\end{tabular} & $\begin{array}{l}\text { - Table des signaux moyennés à } 5 \text { min } \\
\text { - Table événementielle des signaux à } \\
\text { 100ms }\end{array}$ \\
\hline
\end{tabular}

Figure 3 - Représentation schématique du fonctionnement du système d'acquisition qui se décompose en trois principales étapes : (i) la lecture des valeurs envoyées par les différents capteurs, (ii) l'utilisation de ces valeurs pour définir l'activité du torrent et réaliser les tâches requises (données événementielles, prises de vue), (iii) la sauvegarde continue des données à 5 min et une sauvegarde événementielle selon le potentiel d'activité torrentielle (seuil pluviométrique).

Pour suivre l'état de fonctionnement de la station, les données moyennées à cinq minutes sont quotidiennement envoyées par routeur GSM/GPRS puis mises en ligne sur une page web (Figure 4). Cet outil permet à la fois : (i) de suivre l'état de fonctionnement de l'ensemble du dispositif de mesure (centrale d'acquisition et capteurs) pour déceler les potentiels dysfonctionnements du système, et (ii) de surveiller l'activité du torrent à distance pour, si nécessaire, déclencher une campagne de terrain post-crue.

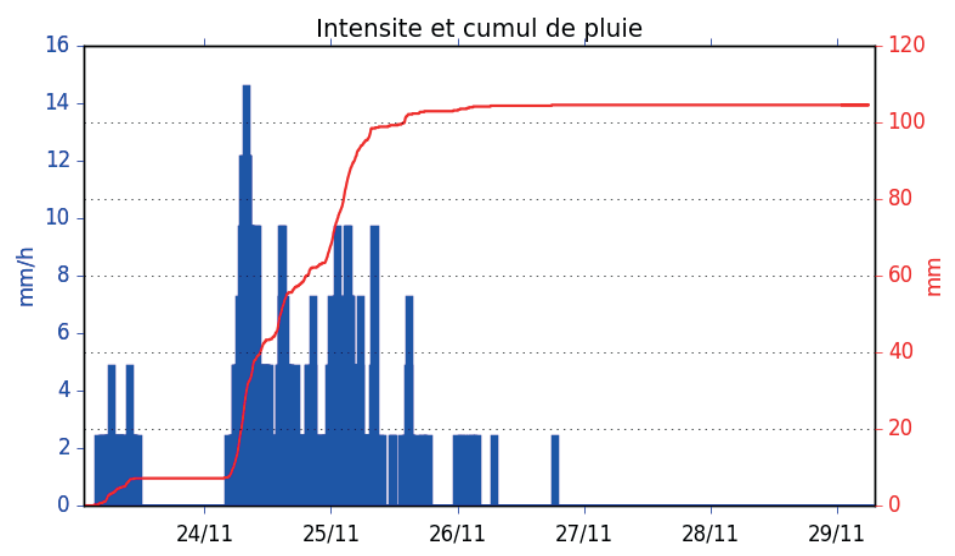

Figure 4 - Exemple de deux graphiques affichés sur le site web : http://monitoringstations.grenoble.cemagref.fr.

En haut : intensité et cumul pluviométrique; en bas : activité sismique en berge du torrent. 


\section{Pluviométrie, mesure de niveau d'écoulement et prises de de vues}

La mesure des précipitations est assurée par un pluviomètre à auget basculant (ARG100 Campbell®). Ce capteur passif est relativement robuste mais nécessite un entretien régulier pour avoir une mesure de qualité (nettoyer les feuilles, pollens, insectes). Lors de son implantation, il est nécessaire de s'assurer que la mesure soit la plus représentative possible des précipitations à l'échelle du bassin versant. N'étant pas pourvus de système chauffant, ces pluviomètres ne sont pas capables de restituer correctement les précipitations solides. Il est donc difficile d'interpréter l'initiation des crues liées à la fonte rapide de neige.

La mesure du niveau d'écoulement est assurée par un capteur non intrusif, basé sur le calcul du temps d'aller-retour d'une série d'échos. Les capteurs ultra-

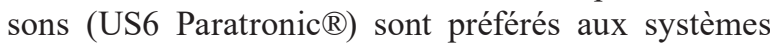
radars dans le cas des torrents car ils ne sont pas affectés par l'absence d'écoulement. L'ultrason est émis selon un cône d'émission de $\pm 6^{\circ}$, ce qui induit une mesure intégrée sur une surface d'environ $0,9 \mathrm{~m}^{2}$ pour un capteur à $5 \mathrm{~m}$ de hauteur. La qualité de l'écho va dépendre de l'état de la surface de réflexion (la meilleure configuration étant une surface horizontale et homogène). Pour pallier cela, il est possible d'augmenter la durée de rafraichîssement de la mesure, i.e. le nombre d'échos sur lesquels le capteur fait une moyenne temporelle. Un compromis sur cette durée de rafraîchissement est donc nécessaire pour conserver une bonne description temporelle de la dynamique de la crue (front raide des laves torrentielles).

En complément, les prises de vues durant la crue fournissent des informations qualitatives très utiles pour l'interprétation des signaux. La centrale d'acquisition, associée à des relais, permet de contrôler à la fois l'alimentation et le déclenchement des appareils. En plus d'un time lapse (un cliché par jour), des prises de vues événementielles sont déclenchées lors des crues grâce à des seuils basés sur l'intensité de précipitation et la sismicité. Le dépassement d'un premier seuil de 'réveil' induit la mise sous tension des appareils normalement éteints pour limiter leur consommation. Suite au dépassement d'un second seuil de 'déclenchement', des clichés sont réalisés toutes les une à dix secondes suivant les capacités des appareils photographiques (plus d'information dans Piton et al., 2017).

\section{Mesure sismique}

Durant les crues transportant des matériaux grossiers (graviers, blocs), la collision et le frottement entre les particules et le lit génèrent des vibrations sismiques dans le sol décelables à proximité du chenal. Ces vibrations se propagent sous la forme d'ondes de surface et couvrent une plage fréquentielle comprise entre 10 et 80-100 Hz (Huang et al., 2007 ; Burtin et al., 2016). Il est possible de capter ces vibrations de manière non intrusive à l'aide de géophones. Plusieurs types d'installation sont possibles : par exemple, les capteurs peuvent être (i) placés dans le lit du torrent, protégés par des plaques ou des tubes, ou (ii) implantés sur les berges du torrent (configuration que nous avons adoptée ; Figure 5b). Caractériser au mieux le signal sismique - en respectant le critère de Shannon - nécessiterait un système d'acquisition haute fréquence (i.e., $\mathrm{kHz}$ ) peu compatible avec le monitoring en montagne (forte consommation énergétique, volume conséquent de données à stocker et à traiter). Pour pallier ce problème, des méthodes de conditionnement des signaux ont été développées (Arattano et al., 2014) et permettent notamment de restituer l'information
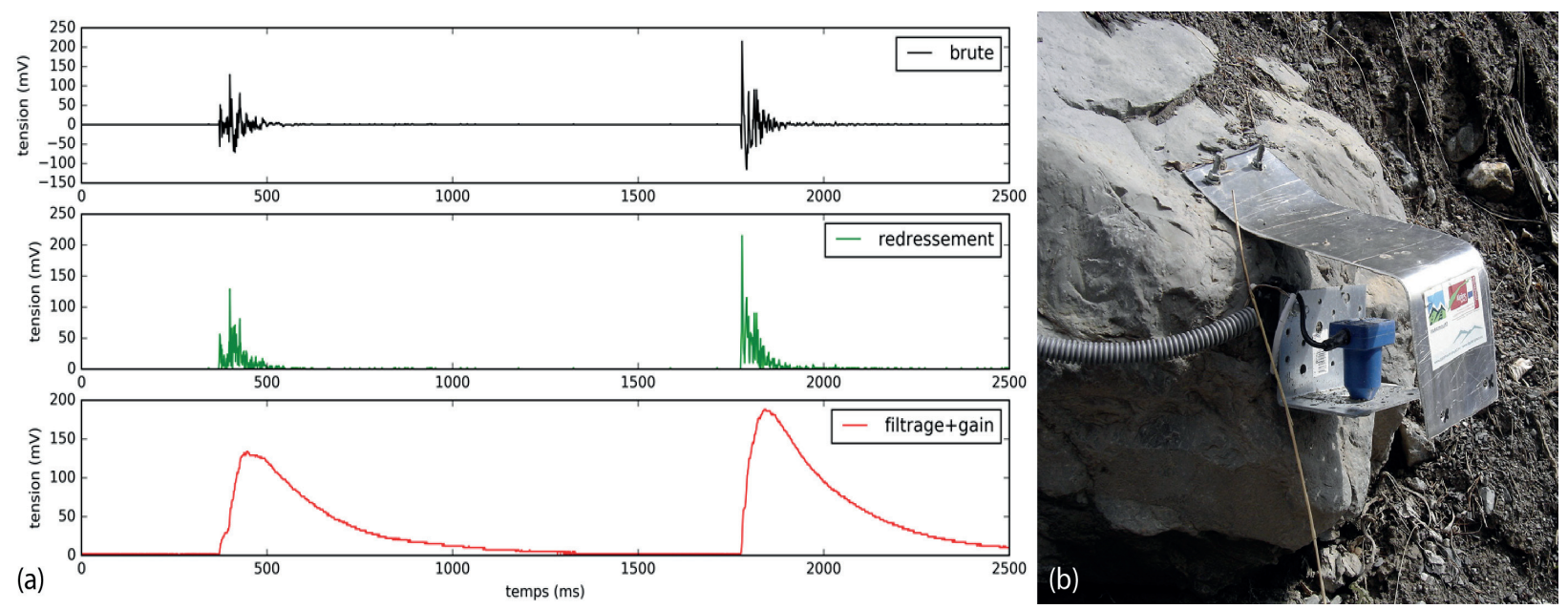

Figure 5 - (a) Conditionnement des signaux géophones. Différentes étapes du traitement analogique : (i) signal initial, (ii) signal redressé et (iii) signal filtré et amplifié. b) implantation d'un géophone (GS20DX Geospace $\left.{ }^{\circledR}\right)$ fixé à un bloc sur la berge du torrent du Manival (38). 
nécessaire à la détection des crues. La première, dite " méthode des impulsions ", consiste à comparer le signal sismique à une valeur d'amplitude arbitraire et à compter le nombre de dépassements du seuil. Le choix de cette valeur peut avoir un impact fort sur la forme du signal traité. Une seconde approche est d'enregistrer uniquement la moyenne des valeurs absolues du signal sur une seconde. Cette méthode, dite « méthode de l'amplitude ", permet une bonne restitution de la dynamique de la crue mais requiert, pour disposer d'un signal pertinent à $1 \mathrm{~Hz}$, une fréquence d'acquisition encore trop importante (e.g., $100 \mathrm{~Hz}$ ).

Les centrales d'acquisition environnementales ne permettent pas de travailler à de telles fréquences. Nous avons donc développé une carte électronique qui reproduit cette opération sur le signal analogique, avant l'échantillonnage par la centrale. Le signal brut en sortie du géophone est (i) redressé, (ii) filtré puis (iii) amplifié avant d'être (iv) transmis, par boucle de courant, à la centrale d'acquisition et (v) échantillonné à une fréquence de $10 \mathrm{~Hz}$ (Figure 5a). L'amplification permet d'éloigner les géophones du chenal et donc d'éviter leur arrachement lors d'une crue. Cependant un compromis gain/distance est à trouver car une trop forte amplification diminue le rapport « signal physique généré par le capteur » sur «bruit électronique généré par la carte », et donc la qualité de la mesure. L'avantage de cette méthode est qu'elle permet de conserver l'énergie du signal initial même avec une faible vitesse d'acquisition.

\section{RÉSULTATS ET APPLICATIONS}

L'usage de stations de monitoring permet de caractériser l'activité torrentielle du bassin versant équipé, en s'intéressant au transport sédimentaire résultant de chaque évènement de pluie (Figure 6 ; Bel, 2017). Il est ainsi possible d'identifier les occurrences d'un phénomène de transport, de connaître la nature de l'écoulement et d'estimer quelques grandeurs physiques telles que le débit de pointe ou le volume d'une lave torrentielle. Ces informations alimentent de façon exhaustive - c'est-à-dire en considérant les évènements de pluie avec et sans transport solide, qu'ils aient ou non engendré des dégâts - une base de données événementielles. La chaîne de traitement proposée (Figure 6) - avec les critères empiriques adéquats - a permis de développer un outil d'analyse semi-automatique pour faciliter la gestion du volume de données. Sur des temps relativement longs (pluriannuels, variables selon les bassins versants), celle-ci permet d'établir des relations empiriques clés lors de l'évaluation de l'aléa torrentiel. Une telle base de données permet ainsi d'évaluer les conditions pluviométriques d'occurrence d'une crue torrentielle, en établissant par exemple, un seuil de pluie probabiliste intensité-durée (Berti et al., 2012).

Lors des épisodes de transport fortement transitoires (lave torrentielle mature ou immature), il est possible d'évaluer la vitesse de propagation d'une bouffée à partir du déphasage entre plusieurs signaux géophones, en recherchant le maximum de corrélation temporelle. Ensuite, connaissant le profil topographique de la section en travers, il est possible d'estimer, en couplant la vitesse aux mesures de hauteur, le débit, le volume d'une bouffée et la magnitude d'un événement (i.e. le volume total incluant parfois plusieurs bouffées).

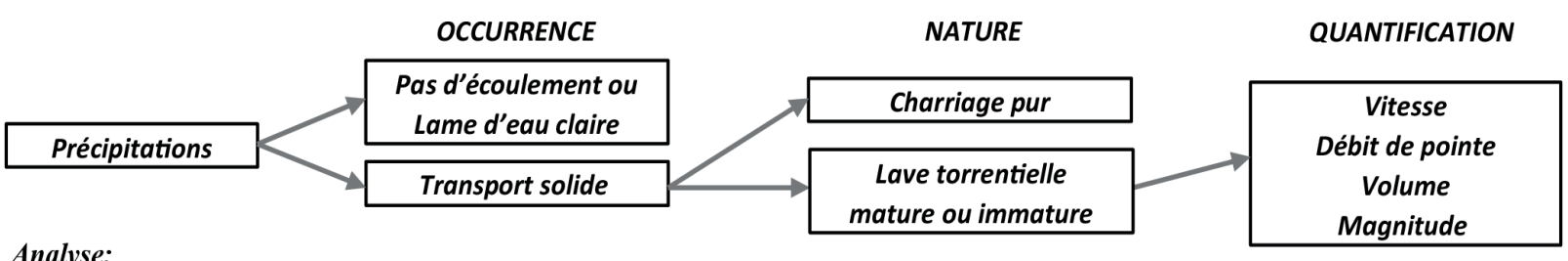

Analyse:

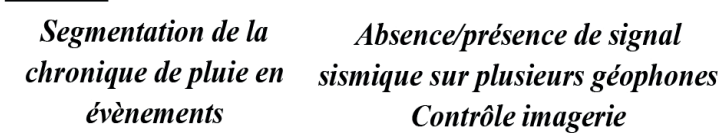

Calculs: intensité, durée

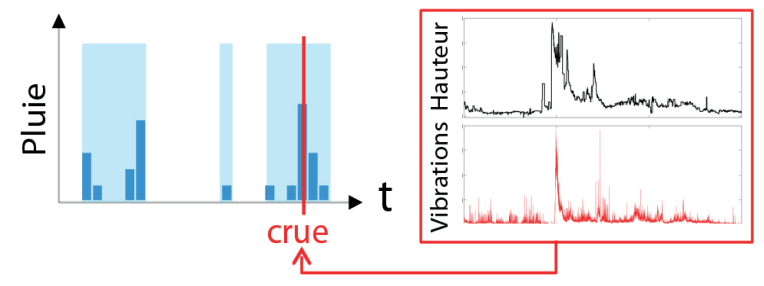

\section{Absence/présence d'un signal fortement transitoire sur plusieurs géophones Contrôle imagerie*}

Applications :

Figure 6 - Synoptique des informations fournies par une station de monitoring : caractérisation de la réponse d'un torrent à un forçage pluviométrique et exemple d'applications de la base de données événementielles, plus de détails dans (Bel, 2017). (*voir Piton et al., 2017).

\section{Calculs: déphasage des signaux sismiques, vitesse, débit, volume}
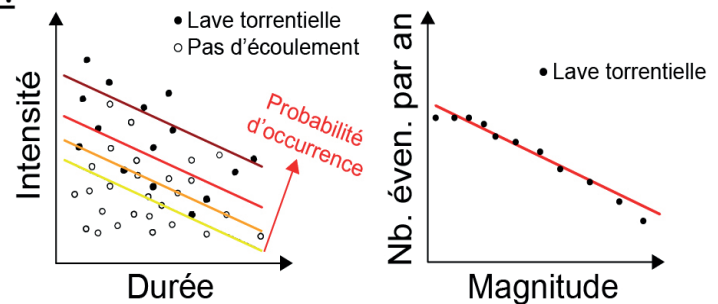


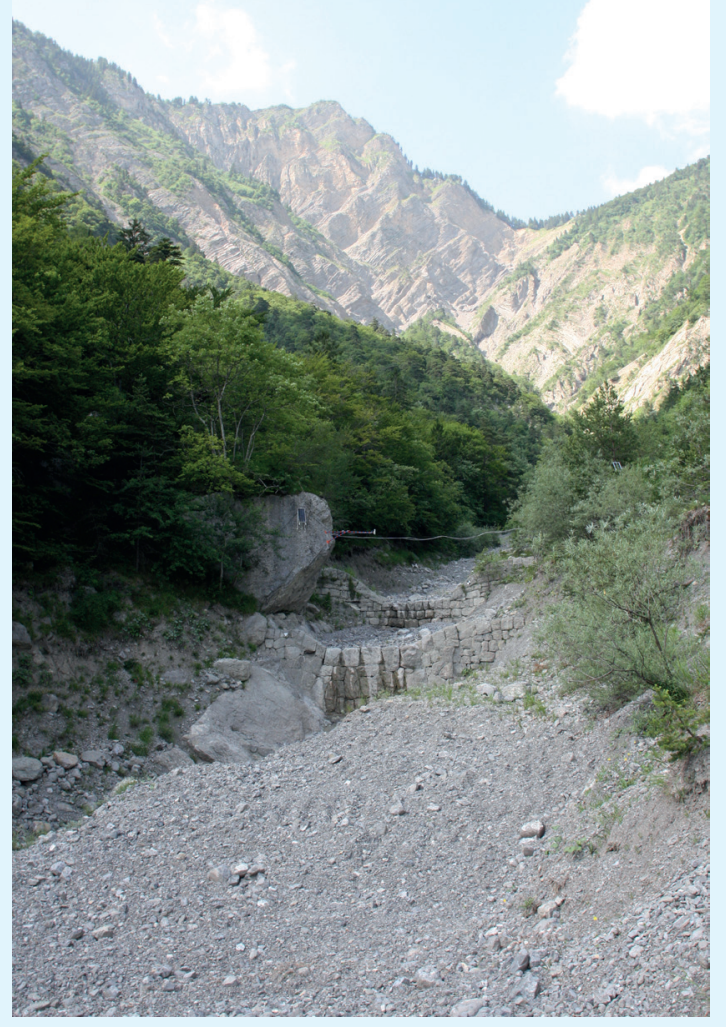

Torrent du Manival (38): seuil instrumenté. Vue vers l'amont, avec les sources sédimentaires en arrière plan.
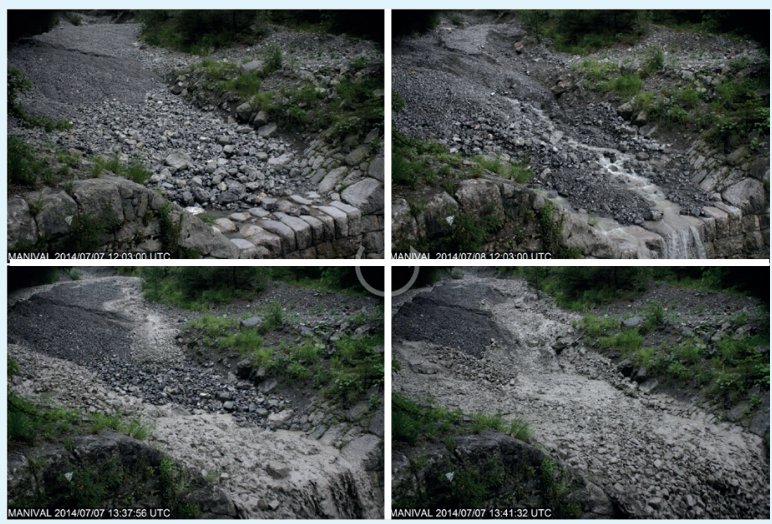

Torrent du Manival (38) : changements morphologiques au cours de la crue du 7 juillet 2014.

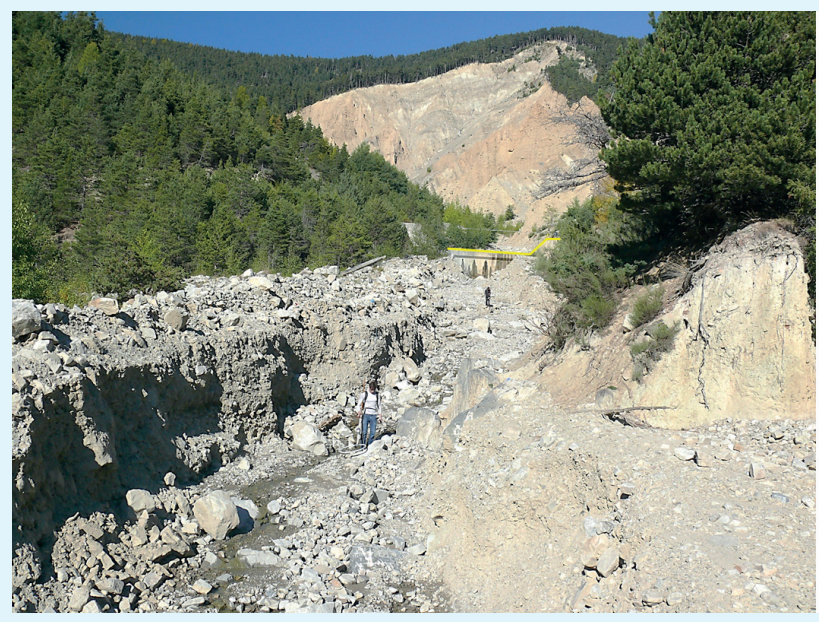

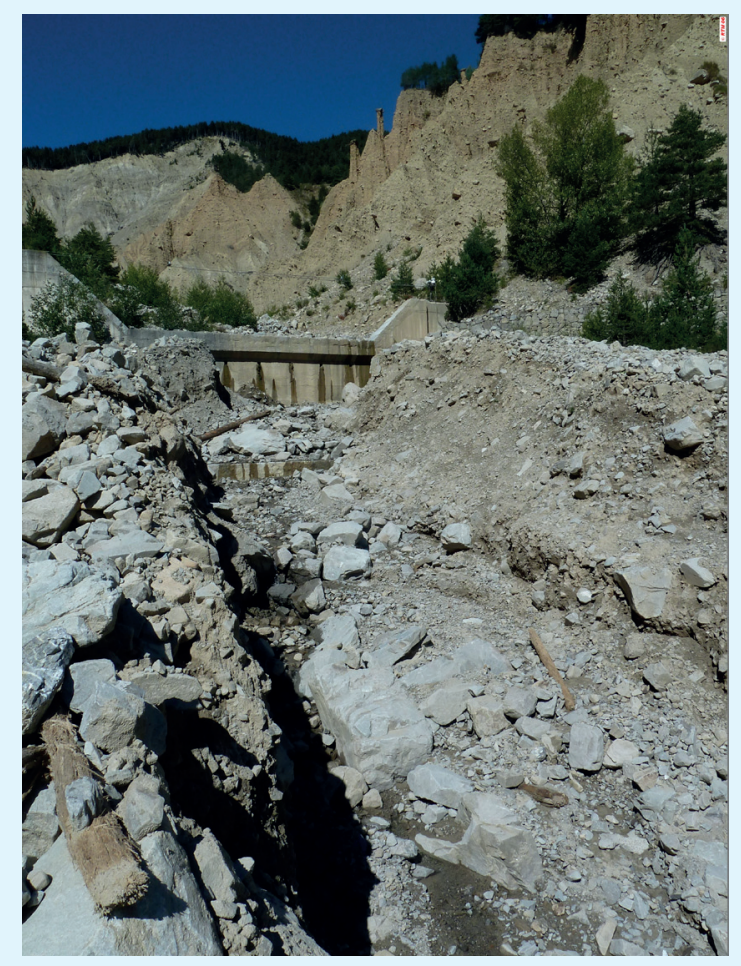

Torrent du Réal (06), partie amont; vue vers l'amont, sources sédimentaires en arrière plan (seuil instrumenté).

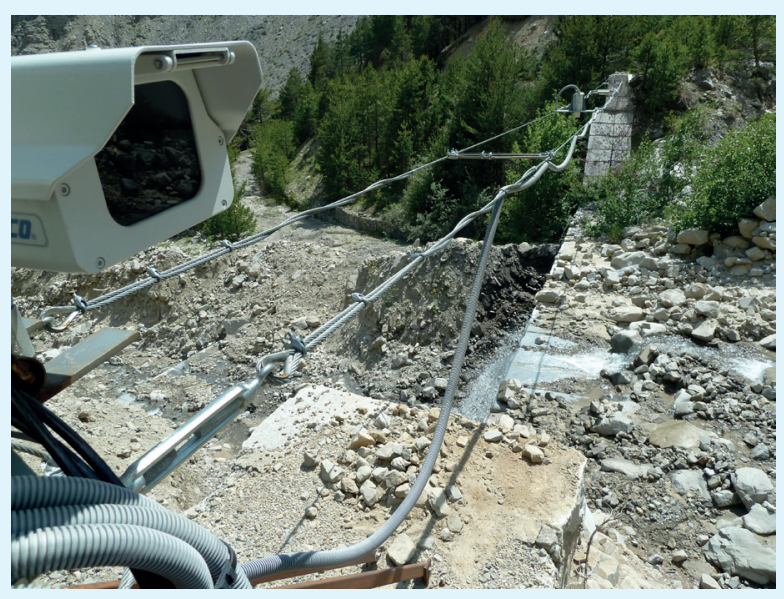

Torrent du Réal (06) : seuil instrumenté sur la partie amont $d u$ torrent (appareil photo et capteur ulstrason).

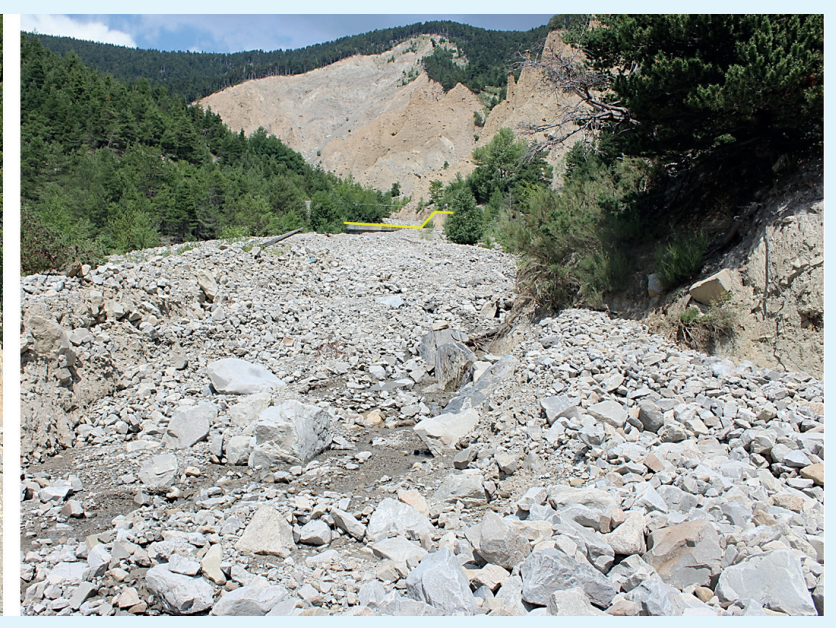

Torrent du Réal (06) : avant-après la crue du 18 juillet 2015 (important dépôt en aval du barrage instrumenté). 
Quelques recommandations pour l'installation des stations peuvent permettre de limiter les incertitudes d'estimation: (i) préférer une distance inter-géophone courte-de l'ordre de $50 \mathrm{~m}$ - pour réduire la possibilité de divagation, se prémunir des changements de nature de l'écoulement et ne pas sous-estimer le débit en étant représentatif de la vitesse locale; (ii) choisir une section contrôlée par un barrage, pour éviter les problèmes d'érosion ou de dépôt faisant fortement varier la section d'écoulement (source principale d'incertitude); (iii) favoriser une section en travers confinée pour s'assurer que l'écoulement occupe toute la section et avoir une mesure de hauteur représentative. Dans ces conditions, on peut s'attendre à des incertitudes de l'ordre de $\pm 30 \%$ sur les volumes estimés. Ces caractéristiques d'écoulement peuvent être utilisées afin d'établir des relations entre la fréquence annuelle d'événements et leur magnitude.
La position de la station de monitoring dans le bassin versant a un impact important sur la caractérisation de l'activité torrentielle. La réponse torrentielle sera d'autant plus complexe que la surface drainée sera importante, car les conditions nécessaires à la propagation de la crue viennent s'ajouter à celles de son déclenchement. En effet, toutes les laves déclenchées en tête de bassin ne se propagent pas jusqu'à la zone d'enjeux à l'exutoire. En plus des prédispositions morphométriques, l'état des stocks sédimentaires, à la fois dans le haut bassin et dans le chenal - qui dépend notamment de la chronique des crues antécédentes - joue un rôle essentiel sur la susceptibilité torrentielle. En plus des contraintes d'accès aux sites viennent s'ajouter des questions de représentativité de ce que l'on souhaite mesurer (petites crues rechargeant le chenal ou fortes crues menaçant les enjeux).

\section{Conclusion}

Les stations de monitoring ont prouvé leur intérêt en matière de caractérisation des épisodes de transport solide en milieu torrentiel, à l'échelle locale. L'usage de tel système est principalement limité par une forte demande de maintenance et un traitement de données lourd. L'émergence d'interfaces de suivi à distance, l'application de modes événementiels et le développement d'outils d'analyse semi-automatiques facilitent aujourd'hui l'intégration de ces systèmes et devraient encourager le développement de nouveaux dispositifs en contexte opérationnel.

\section{Remerciements}

Nous remercions les financeurs de ces recherches : le projet Interreg Espace Alpin Sedalp, les projets Interreg Alcotra TTCoCo et Risknat, le projet Captiven et enfin IRSTEA. Un remerciement particulier est adressé à G. Astier (RTM05), I. Chouquet (CD05) et $R$. Gaucher (CD05) pour l'aide qu'ils ont pu apporter sur le terrain et lors du montage de projets, ainsi qu'à F. Ousset, X. Ravannat et C. Eymond-Gris pour leur aide à la conception et à l'installation des dispositifs.

\section{Bibliographie}

Arattano M., Abancó C., Coviello V., Hürlimann M., 2014. Processing the ground vibration signal produced by debris flows: the methods of amplitude and impulses compared. Computers \& Geosciences, 73, 17-27.

Badoux A., Graf C., Rhyner J., Kuntner R., McArdell B., 2009. A debris-flow alarm system for the alpine Illgraben catchment: design and performance. Natural Hazards, 49 (3), 517-39.

BEL C., 2017. Analysis of debris-flow occurrence in active catchments of the French Alps using monitoring stations. $P h D$. PhD thesis, Univ. Grenoble Alpes, $186 \mathrm{p}$.

Berti M., Martina M.L.V., Franceschini S., Pignone S., Simoni A., Pizziolo M., 2012. Probabilistic rainfall thresholds for landslide occurrence using a Bayesian approach. Journal of Geophysical Research, 117, 20 p.

Burtin A., Hovius N., Turowski J.M., 2016. Seismic monitoring of torrential and fluvial processes. Earth Surface Dynamics, 4 (2), 285-307.

Cavalli M., Marchi L., Arattano M., Comiti F., Schenato L., Liébault F., 2013. Protocol for debrisflow monitoring. In Interreg Alpine Space Sedalp Project, Technical report, WP5 Protocol for data collection method in sediment transport, Part II, 36-p. (www.sedalp.eu)
Huang C.J., Yin H.Y., Chen C.Y., Yeh C.H., Wang C.L., 2007. Ground vibrations produced by rock motions and debris flows. Journal of Geophysical Research, 112, 20 p.

Navratil O., Liébault F., Bellot H., Theule J., Ravanat X., Ousset F., Laigle D., Segel V., Fiquet M., 2011. Installation d'un suivi en continu des crues et laves torrentielles dans les Alpes françaises. In Journée de Rencontre sur les Dangers Naturels, Institut de Géomatique et d'Analyse du Risque, Lausanne, Suisse, $8-\mathrm{p}$.

Piton G., Berthet J., Bel C., Fontaine F., Bellot H., Malet E., Astrade L., Liebault F., Recking A., Juppet M., Royer K., 2017. Dynamique géomorphologique des torrents : intérêt de l'emploi des appareils photographiques automatiques. In Malet E., Astrade L., Monitoring en milieux naturels - Retours d'expériences en terrains difficiles, Collection Edytem, n¹9, 203-210.

Recking A., Richard D., Degoutte G., 2013. Torrents et rivières de montagne : Dynamique et aménagement. Quae Edition, 336-p. 
